\title{
DETERMINACIÓN DE LA ACTIVIDAD FÍSICA EN EL RECREO ESCOLAR: COMBINANDO MEDICIONES DE ACTIVIDAD FÍSICA Y LA PERSPECTIVA ESTUDIANTIL
}

Álvarez Bogantes, Carlos; Villalobos Víquez, Grettel; Vargas Tenorio, Jennifer DETERMINACIÓN DE LA ACTIVIDAD FÍSICA EN EL RECREO ESCOLAR: COMBINANDO MEDICIONES DE ACTIVIDAD FÍSICA Y LA PERSPECTIVA ESTUDIANTIL

MHSalud, vol. 14, núm. 2, 2018

Universidad Nacional, Costa Rica

Disponible en: http://www.redalyc.org/articulo.oa?id=237054293004

DOI: https://doi.org/10.15359/mhs.14-2.4

Esta obra está bajo una Licencia Creative Commons Atribución-NoComercial-SinDerivar 3.0 Internacional. 


\title{
DETERMINACIÓN DE LA ACTIVIDAD FÍSICA EN EL RECREO ESCOLAR: COMBINANDO MEDICIONES DE ACTIVIDAD FÍSICA Y LA PERSPECTIVA ESTUDIANTIL
}

\author{
DETERMINATION OF PHYSICAL ACTIVITY DURING SCHOOL RECESS COMBINING \\ MEASUREMENTS OF PHYSICAL ACTIVITY AND CHILDREN'S PERSPECTIVE \\ DETERMINAÇÃO DA ATIVIDADE FÍSICA NO RECREIO ESCOLAR: COMBINANDO \\ MEDIÇÕES DE ATIVIDADE FÍSICA E A PERSPECTIVA ESTUDIANTIL
}

\begin{abstract}
Carlos Álvarez Bogantes ceab.03@gmail.com
Universidad Nacional, Costa Rica

Grettel Villalobos Víquez grevv26@gmail.com

Universidad Nacional, Costa Rica
\end{abstract}

Jennifer Vargas Tenorio jennifervt3@gmail.com

Universidad Nacional, Costa Rica

Recepción: 21 Noviembre 2017

Aprobación: 26 Enero 2018

DOI: https://doi.org/10.15359/mhs.14-2.4

Redalyc: http://www.redalyc.org/articulo.oa?id=237054293004

\begin{abstract}
Resumen:
El objetivo de este estudio fue determinar los niveles de actividad física de los estudiantes durante el recreo escolar, tomando en cuenta las percepciones de los estudiantes y observaciones del recreo escolar. Se aplicó un método mixto, utilizando el instrumento de observación de juego y actividades recreativas (SOPLAY) y entrevistas. Los participantes fueron estudiantes de tres escuelas públicas de horario ampliado y alterno, que fueron observados en los recreos escolares. Adicionalmente, se realizaron grupos focales. Se utilizó estadística descriptiva, y para determinar si existieron diferencias entre niveles de actividad física moderada-vigorosa entre tipos de escuela, se realizó una prueba de varianza factorial (2x2). Los resultados de los grupos focales se organizaron en categorías. Los estudiantes de este estudio presentaron 47.98 de actividad sedentaria, con un 52.02 de actividad física moderada vigorosa en el recreo escolar, siendo las niñas más sedentarias que los niños. Los escolares de escuelas que alternan son más activos que los de escuelas de horario ampliado. Las personas participantes percibieron que las barreras para la realización de actividad física tienen que ver con espacio reducido para jugar, con poco apoyo de las maestras. En conclusión, este estudio mostró que un poco más de 50\% de los estudiantes realizan actividad física en los recreos escolares, siendo los niños y niñas de escuelas de horario alterno más activos que los de horario ampliado. Las personas participantes perciben que los entornos escolares no favorecen la actividad física, debido a las barreras ambientales. Con base en los resultados de este estudio, se recomienda promover la actividad física a través del recreo escolar, considerando las barreras en el entorno natural, social, físico y organizativo.
\end{abstract}

Palabras clave:

Actividad física, Barreras, Niñez, Recreo Escolar, Percepción, tipos de escuela.

\section{Abstract:}

The aim of this study was to determine physical activity levels of children during school recess, taking into account children's perceptions as well as observations during recess. A mixed method was used, including the System for Observing Play and Leisure Activity in Youth (SOPLAY) and focus groups. Participants included students from 3 public schools with regular and alternating schedules, who were observed during their school breaks. In addition, focus groups were held and descriptive statistics were used. A factorial variance test $(2 \times 2)$ was also used to determine if there were differences between levels of moderate-vigorous physical activity among school types. Results of focus groups were organized into 
categories. Students exhibited 47.98 sedentary activity and 52.02 moderate-vigorous physical activity during school recess, with girls being more sedentary than boys. Students with an alternating schedule are more active than those with a regular schedule. Participants perceived reduced space to play and little support from teachers as barriers to do physical activity. In conclusion, this study showed that a little over $50 \%$ of students perform physical activity during school recess, with children in alternating schedules being more active than those with a regular schedule. Participants perceive that school environment does not favor physical activity, due to environmental barriers. Based on the results of this study, physical activity should be promoted during school recess, taking into consideration barriers in natural, social, physical, and organizational environments.

\section{Keywords:}

Physical activity, Barriers, Childhood, School Recess, Perception, Types of School.

Resumo:

O objetivo deste estudo foi determinar os níveis de atividade física dos estudantes durante o recreio escolar, levando em consideração as percepções dos estudantes e observações do recreio escolar. Aplicou-se um método misto, utilizando o instrumento de observação de jogo e atividades recreativas (SOPLAY) e entrevistas. Os participantes foram estudantes de três escolas públicas de horário ampliado e alternado, que foram observados nos recreios escolares. Além disso, foram realizados grupos focais. Utilizou-se estatística descritiva, e para determinar se existiram diferenças entre níveis de atividade física moderada-vigorosa entre tipos de escola, aplicou-se um teste de variância fatorial (2×2). Os resultados dos grupos focais foram organizados em categorias. Os estudantes deste estudo apresentaram 47,98 de atividade sedentária e 52,02 de atividade física moderada vigorosa no recreio escolar, sendo as meninas mais sedentárias do que os meninos. Os estudantes de escolas que alternam seu horário são mais ativos do que os de escolas de horário ampliado. As pessoas participantes perceberam que as barreiras para a realização de atividade física têm a ver com o espaço reduzido para brincar e com o pouco apoio das professoras. Em conclusão, este estudo mostrou que um pouco mais de $50 \%$ dos estudantes realizam atividade física nos recreios escolares, sendo os meninos e meninas de escolas de horário alternado mais ativos do que os de horário ampliado. As pessoas participantes percebem que os entornos escolares não favorecem a atividade física, devido às barreiras ambientais. Com base nos resultados deste estudo, recomenda-se promover a atividade física através do recreio escolar, considerando as barreiras no entorno natural, social, físico e organizacional.

Palavras-chave:

Atividade física, Barreiras, Infância, Recreio Escolar, Percepção, tipos de escola.

\section{Introducción}

En la actualidad, Costa Rica no escapa a la epidemia del sobrepeso y obesidad, experimentando un incremento de la incidencia y prevalencia de sobrepeso y obesidad infantil. Según el Ministerio de Salud y el Ministerio de Educación Pública (2016), en el censo escolar de peso y talla se estimó que el sobrepeso y la obesidad de los escolares costarricenses es de 34\%. Aunado a lo anterior, los niveles de sedentarismo se han convertido en uno de los problemas de salud más críticos (Organización Panamericana de la Salud [OPS], 2010). Fuster (2014) ha indicado que el sedentarismo se relaciona con el incremento de factores de riesgo cardiovascular, asociado a la obesidad y a enfermedades crónicas no transmisibles. En nuestro país se han reportado niveles alarmantes de sedentarismo de un $27.4 \%$ en la niñez (Instituto de Nutrición de Centroamérica y Panamá [INCAP], 2016).

El incremento en los niveles de sedentarismo, la falta de oportunidades de actividad física y el incremento de la niñez con sobrepeso y obesidad en todo el mundo (Janssen y Leblanc, 2010) han llevado a considerar las escuelas como los espacios fundamentales para que los escolares puedan ser activos y construyan las destrezas necesarias para enfrentar una cultura sedentaria (Pawlowski, Tjørnhøj-Thomsen, Shipperijn y Troelsen, 2014).

La OMS (2010) identificó también a estos establecimientos como esenciales para contribuir a cumplir la hora de actividad física de moderada-vigorosa intensidad recomendada como estrategia para combatir el sobrepeso y la obesidad en los infantes, ya que es en estos entornos donde ellos permanecen la mayor cantidad de tiempo durante el día, pudiéndose dar una gran prioridad a la práctica de la actividad física 
regular como herramienta para la prevención de la obesidad infantil en el contexto escolar, con el fin de propiciar un abordaje temprano que optimice los resultados y el impacto en la salud (Davidson y Lawson, 2006; Biddle y Asare, 2011). Dentro de dicho contexto, el recreo es un espacio importante en el camino de lograr la meta de tener una población escolar activa. Se ha reconocido que el recreo presenta el potencial de poder contribuir hasta con un $40 \%$ de los 60 minutos diarios recomendados de actividad física para los estudiantes, bajo las condiciones apropiadas (Frago, Murillo, García, Aibar y Zaragoza, 2017).

De acuerdo con estudios previos cuantitativos realizados en otros países, la proporción de tiempo dedicado a la actividad física de moderada a vigorosa intensidad durante la mañana y el recreo del almuerzo es de 32,1\% en los varones y 23,7\% en las niñas, en Francia (Blaes et al., 2013), y de 32,9\% en los varones y $25,3 \%$ en las niñas, en Inglaterra (Ridgers, Stratton, 2005). En el entorno costarricense se carece de la información acerca de los niveles de actividad física de los escolares en los ambientes de recreo, por lo que se hace necesario no solo conocer el grado de actividad física de los escolares en ese entorno, sino también sus percepciones, las cuales brinden mayores elementos para entender la conducta de movimiento, con el fin de poder desarrollar intervenciones que respondan a la realidad escolar costarricense. Adicionalmente, es importante recalcar que la influencia de los factores ambientales sobre la actividad física durante el recreo difiere de acuerdo con la cultura y con los estilos de vida de los países, según estudios cualitativos desarrollados en diferentes culturas (Ishii, Shibata, Sato y Koichiro, 2014; Haug, Torsheim, Sallis y Samdal, 2010), lo que hace necesario indagar estos factores en el entorno costarricense desde la perspectiva de los estudiantes.

En el entorno escolar costarricense, especialmente en el espacio del receso escolar, se desconocen las conductas, patrones y percepciones de los escolares acerca de actividad física. Contar con dicha información es necesario para poder tener no solo un conocimiento de la problemática que se enfrenta en cuanto a los niveles de actividad física de los escolares, sino también para poder desarrollar propuestas y políticas que promuevan una cultura en movimiento en los recreos escolares. Respondiendo al llamado de salud pública de asumir un rol activo en promover y gestar modelos para la promoción de la salud escolar, especialmente en la búsqueda de combatir problemas como el sedentarismo, la obesidad, las enfermedades cardiovasculares y la diabetes tipo II (Hayman et al., 2004), este estudio brindará los insumos iniciales requeridos para poder desarrollar una propuesta de recreos saludables en el entorno costarricense desde la realidad cotidiana escolar y la voz de los estudiantes.

Este tipo de estudio se ha reconocido como la combinación perfecta para profundizar en el entendimiento y obtención de información directa sobre el entorno de los recreos escolares en la promoción de la actividad física de los escolares. Como se ha establecido, se requiere hacer partícipes a los escolares a través de escucharlos y entenderlos en la búsqueda del entendimiento de sus percepciones, creencias y actitudes hacia el movimiento humano y de sus barreras en la construcción de una cultura más saludable (Pawlowski, Tjørnhøj-Thomsen, Shipperijn y Troelsen, 2014).

El objetivo de este estudio es determinar los niveles de actividad de los niños en los espacios de juego en el entorno escolar durante los recreos, incorporando un acercamiento cualitativo a las percepciones de estos acerca de las barreras para ser activos físicamente.

\section{Metodología}

\section{Participantes}

Este estudió contempló la observación de 90 niños y niñas de primer ciclo y segundo ciclo, durante los recreos escolares en una escuela pública de horario ampliado y dos escuelas de horario alterno, de atención prioritaria del cantón Central de Heredia, seleccionadas al azar. En escuelas de horario ampliado los estudiantes asisten a lecciones de 7 a.m. a 1:30 p.m. todos los días, y en las escuelas donde se alterna, los estudiantes van de 7 a 12 md. En estas escuelas, los de un ciclo asisten unos días por la mañana, mientras que los de el otro ciclo lo hacen por la tarde. En la parte cualitativa de este estudio, se realizaron dos grupos focales con 12 niños y niñas de cada institución. 


\section{Instrumento}

En la parte cuantitativa de esta investigación se utilizó el protocolo o sistema de observación del juego y tiempo libre (SOPLAY). Esta es una herramienta validada usando monitores cardiacos (McKenzie, Sallis y Nader, 1991), que ofrece correlaciones altas para las niñas sedentarias $(R=.98)$ y las niñas que caminaban (.95), aunque menor para los recuentos de las chicas muy activas (.76). Para los niños, las correlaciones fueron altas para sedentarios (.98), caminar (.98) y comportamiento muy activo (.97). Todos los resultados entre observadores y las correlaciones intraclase cumplieron criterios aceptables $(I O A=80 \%, R=.75)$ para una evaluación confiable.

SOPLAY aporta datos objetivos sobre el número de participantes y sus niveles de actividad física durante espacios de juego y de ocio. Este protocolo consiste en observar o hacer escaneos de la intensidad y actividad física que realizan los niños en cada una de las áreas de juego de la institución por 15 segundos, en cada uno de los recreos institucionales, observando las áreas de izquierda a derecha y anotando esencialmente los niveles de actividad física de los niños observados, según cada área señalada en la escuela. Durante un escaneo, la actividad física da cada escolar en un área es codificada como sedentaria (sentado o de pie), actividad física de moderada intensidad (incluyendo caminata) o actividad física vigorosa. Las observaciones se hicieron dos veces en dos días típicos de los recreos escolares, con el objeto de corroborar los resultados.

Para la conducción de los grupos focales, se utilizó un cuestionario con preguntas abiertas a los estudiantes de los grupos participantes. Este cuestionario se construyó con base en la revisión bibliográfica sobre la temática de recreos, los objetivos propuestos en la investigación y la experiencia de los investigadores, siguiendo el modelo socioecológico (Ward y Saunder, 2007).

\section{Procedimiento}

Se contó con la aprobación del Ministerio de Educación y con la anuencia del director de cadainstitución educativa; luego, se procedió a observar informalmente los recreos para determinar las áreas de juego de los escolares, medirlas y preparar las condiciones para las observaciones. Una vez realizado el mapeo de las áreas, se procedió a observar los recreos de 10 y 15 minutos durante tres momentos diferentes en cada una de las escuelas participantes. Una vez terminada cada observación, se realizaron los cálculos de los niveles de actividad física de los escolares observados siguiendo las indicaciones dadas por McKenzie, Sallis y Nader (1991).Una vez que se concluyó la fase de observación y con los resultados obtenidos, se procedió a realizar grupos focales con diferentes niveles de los estudiantes de las escuelas participantes, con el objeto de contribuir a un mejor entendimiento de los resultados desde la perspectiva de los observados.

Para la parte cualitativa de esta investigación, se obtuvo el consentimiento informado de los padres de los participantes. Cada sección del primer ciclo de educación primaria seleccionada fue dividida por la orientadora de la institución en cuatro grupos focales mixtos. Las sesiones del grupo focal fueron realizadas en un lugar privado de la institución durante las horas de clase y fueron conducidas por el investigador, con la ayuda de la orientadora de la institución y la maestra de grado. La maestra y un asistente de investigación grabaron y tomaron apuntes de las sesiones, con el fin de fortalecer el análisis posterior de la información y lograr la triangulación necesaria (Patton, 2002). Cada una de las sesiones duró de 30 a 40 minutos. En ellas, primero se explicó la actividad y luego siguió el período de las entrevistas grupales, con un final abierto para la intervención de alguna anécdota que alguno de ellos quisiera compartir.

Para la conducción de los grupos focales se utilizó una entrevista semiestructurada usando preguntas generadoras que emanaron esencialmente del Modelo Socioecológico, con el siguiente orden: 1) preferencias de actividades de los niños en los recreos, 2) factores que motivan a los niños a participar en el juego activo, 3) factores que limitan el juego activo de los niños, y 4) factores que facilitan el juego activo de los niños. 
Diseño

Se utilizó un diseño de método mixto, con una secuencia de etapas cuantitativa, usando el instrumento de observación SOPLAY, seguida de una etapa cualitativa con grupos focales, que se complementan y triangulan, con el fin de lograr un mejor entendimiento, profundización o transformación del fenómeno de estudio (Pereira, 2011).

\section{Análisis de datos}

En relación con la sección cuantitativa de este estudio, se realizó la estadística descriptiva calculando los promedios y las desviaciones estándar de la actividad física. Con respecto a la intensidad de la actividad de los participantes en los recreos se realizó un ANOVA entre la variable dependiente en relación con el género y el tipo de escuelas (escuelas de horario ampliado y de naturaleza alterna).

En la sección cualitativa de este estudio se realizó el proceso de análisis de datos configurado en torno a tres aspectos clave: reducción de datos, disposición y transformación de estos y, por último, la obtención de resultados y la verificación de conclusiones, según lo presentado por Pitney y Parker (2009).

\section{Resultados}

\section{Fase cuantitativa}

La Tabla 1 muestra los promedios y desviaciones estándar de los diferentes niveles de actividad física en forma general y por sexo obtenidos durante los recreos escolares utilizando SOPLAY en las tres escuelas participantes. Se observa que un $47 \%$ de los estudiantes observados son sedentarios, $21 \%$ realizan actividad moderada y un $30 \%$ realizan actividad vigorosa. Los valores mencionados indican que el casi el $50 \%$ de los escolares presentan conductas sedentarias. Cuando se observa los resultados entre los niños y las niñas, se observa que los niños (65\%) son más activos que las niñas (38\%) por mucho, cuando se utiliza los valores integrados actividad física de intensidad moderada a vigorosa (MVPA).

Tabla 1

Promedios de la actividad física en los estudiantes de las escuelas observadas durante el recreo

\begin{tabular}{cccc}
\hline Nivel de actividad física & Promedio total (DE) & Niñas (DE) & Niños (DE) \\
\hline Sedentarismo & $47.98(3.2)$ & $61.23(3.7)$ & $34.48(2.4)$ \\
AF Moderada & $21.58(1.9)$ & $24.31(1.9)$ & $18.81(2.0)$ \\
AF Vigorosa & $30.43(3.4)$ & $14.46(1.1)$ & $46.71(4.6)$ \\
MVPA & $52.02(2.7)$ & $38.77(1.6)$ & $65.52(3.5)$ \\
\hline
\end{tabular}

AF: actividad física, MVAF actividad física de moderada intensidad más actividad física vigorosa, (DE): desviación estándar.

En la Tabla 2 se presentan los resultados observados de la actividad física realizada en las escuelas de horario ampliado y las escuelas de horario alterno. Estas últimas presentaron un 61.3\% de MVPA y las escuelas de horario, un $22.6 \%$. Se muestra que los infantes de escuelas de horario ampliado presentan más conductas sedentarias que los de horario alterno. Este hallazgo muestra posibles líneas de cambio en los entornos escolares, que transformen esta debilidad en una fortaleza en cuanto a la promoción de estilos en movimiento en el recreo. 
Tabla 2

Promedio de actividad física según el tipo de horario de las escuelas

\section{Nivel de actividad física}

\section{Escuela de horario} alterno (DE)

\section{Escuela de horario ampliado (DE)}

$77.4(4.3)$

$9.0(0.7)$

$13.5(1.1)$

$22.6(0.91)$

AF: actividad física, MVAF actividad física de moderada intensidad más actividad física vigorosa, (DE): desviación estándar.

Por medio de la prueba de ANOVA $2 \times 2$ se encontraron diferencias significativas para los efectos principales de género $(p<0.017)$ y el tipo de escuela $(p<0.0001)$, sobre el nivel de actividad física MVPA en los recreos escolares; sin embargo, no se encontró una interacción significativa entre las variables estudiadas. Independientemente, tanto el género como el tipo de escuela causaron una diferencia significativa en los niveles MVPA; sin embargo, ambas variables no interactuaron para producir un efecto mayor.

\section{Fase cualitativa}

Se definieron cuatro categorías principales de análisis a la hora de entender los resultados obtenidos tras observar a los escolares durante el recreo: el factor individual, el entorno social, el medio físico y los factores de la organización y de políticas escolares, siguiendo los planteamientos de una visión ecológica (Ward, Saunders y Pate, 2007).

Los niños en general indicaron su afición por el fútbol, mientras que las niñas de niveles superiores mencionaron gran satisfacción por las actividades sedentarias, con un gran componente social con grupos pequeños de compañeras, como lo manifestó la niña 3: "Lo que más disfruto es hablar con mis amigas". Contrario a las niñas mayores de los grupos focales, un $70 \%$ de las niñas de primer grado se mostraron atraídas por juegos de perseguir, como "la anda". Una niña expresó: "Yo tengo que llegar primero al planché para no perder nada del recreo".

Las docentes han sido percibidas por un $68 \%$ de los niños como una barrera, debido a la constante intromisión de ellas en sus juegos. La expresión siguiente es un ejemplo de esta situación: "Ellas no nos dejan jugar bola en los recreos". Sin embargo, las maestras le ofrecen a las niñas pequeñas un gran sentido de seguridad, como expresó una niña: "Con la niña se van los matones".

En la estructura escolar de los recreos, el cuido está en manos de las maestras, función que tiene el énfasis de evitar que los niños no se maltraten; sin embargo, los niños sí manifiestan cierta persecución con respecto a no permitirles jugar algunos juegos. Uno de los niños expresó: "Las profes no nos dejan jugar y nos quitan los trompos".

Por otro lado, los mayores ven a los más pequeños como obstáculos que interrumpen la "mejenga" y se ponen en el camino de ellos: "Generalmente, tenemos que dejar de jugar hasta que los corremos", expresó un niño de Segundo Ciclo.

En el entorno escolar las barreras físicas más frecuentemente mencionadas por los estudiantes para jugar en los recreos son: la falta de instalaciones y la falta de espacio para jugar. No obstante, también las políticas y lugares de juego no son parte de las preocupaciones de la administración escolar y depende algunas veces de la maestra el ignorar alguna disposición que prohíbe jugar "bola" y les permite jugar a los niños a sus anchas. 


\section{Discusión}

Los hallazgos de este estudio resaltan el gran aporte ofrecido por los métodos mixtos utilizados en esta investigación, en el proceso de entender la actividad física de los escolares y su percepción sobre las barreras que les impiden ser activos. Esta situación en particular hace del recreo no solo uno de los principales momentos en el contexto escolar para la promoción de estilos de vida activos, sino también uno de los principales aliados en la lucha contra el sobrepeso y la obesidad en la niñez costarricense. El recreo escolar ha mostrado ser apropiado para la práctica de actividad física (AF) de forma libre o dirigida; sin embargo, los resultados de este estudio muestran que la conducta que prevalece entre los escolares es el sedentarismo, encontrándose que ellos perciben que el entorno escolar durante los recreos no favorece la actividad física.

Los valores encontrados en esta investigación indican que casi el 50\% de los escolares presentan conductas sedentarias. En este estudio se pudieron observar niveles de sedentarismo mucho más altos que los observados en previos estudios en otros entornos (Anthamatten et al., 2011; Blaes et al., 2013; McKenzie, Marshall, Sallis y Conway, 2000; Springer, Tanguturi, Ranjit, Skala y Kelder, 2013).

La escuela es el lugar donde los infantes pasan la mayor cantidad de tiempo durante la semana, por lo que se considera que los espacios de juego ofrecen el mayor potencial para contribuir a que la población infantil realice la mayor cantidad de actividad física, que les ayude a lograr la recomendación de una hora diaria de actividad física, de moderada a vigorosa (OMS, 2010). Sin embargo, los resultados de este estudio muestran que los participantes presentan conductas sedentarias en los recreos, anulando estos espacios como precursores del movimiento en la niñez.

En el camino de encontrar elementos para explicar los niveles tan altos de sedentarismo que se encontraron en los estudiantes de primaria, se tiene que volver la mirada a aquello que los estudiantes perciben como posibles barreras para su participación en actividades físicas durante los recreos. Los escolares de este estudio consideraron la falta apoyo social como una barrera importante, siendo este nivel de influencia el más significativo a edades tempranas para garantizar el involucramiento en actividades físicas (Mays, Graber y Daum, 2012), lo que hace de suma importancia entender que el recreo es una extensión de la experiencia académica de las aulas, por lo que se recomienda que los estudiantes tengan las condiciones físicas y el apoyo social necesario para que puedan obtener el máximo beneficio de ambientes que estimulan el juego y la actividad física, así como la mayor cantidad de posibilidades para que jueguen (Ickes, Ervin y Beighle, 2013).

Aunque en este estudio la labor de la maestra durante el recreo fue percibida por los estudiantes como una barrera, el aumento en la supervisión de la maestra, en particular cuando ellas participan en el juego, brinda mayor satisfacción y participación por parte de las niñas, propiciando una reducción de conflictos y menos dominación por parte de los niños, como lo han expresado varios estudios (Ozdemir y Yilmaz, 2008; Sallis et al., 2001).

Los ambientes físicos de las escuelas del Valle Central en Costa Rica, donde los estudiantes invierten el tiempo de recreo, carecen de los elementos necesarios para promover movimiento. Como lo ha expuesto Arias (2014) lo reducido de los espacios disponibles para la actividad física durante el recreo refleja la gran cantidad de niños que asumen conductas sedentarias, unido a las políticas escolares de restringir el movimiento en los estudiantes, por razones de seguridad.

La disponibilidad de implementos deportivos durante los descansos ha demostrado ser un factor de motivación para incrementar el nivel de actividad de los escolares (Verstraete, Cardon, De Clercq y De Bourdeaudhuij, 2006). Sin embargo, la falta de espacio disponible para jugar, manifestado por los alumnos de este estudio, y la carencia de implementos para jugar hacen del entorno escolar en un lugar que requiere ser intervenido para cambiar esa situación y crear las condiciones para que la parte administrativa contribuya propiciar ambientes activos (Anthamatten et al., 2011).

Cuando se observan los resultados entre los niños y las niñas, se observa que los niños (65\%) son más activos que las niñas (38\%), por mucho, cuando se utiliza los valores integrados de actividad física de 
intensidad moderada a vigorosa (MVPA). Elliot, Combs y Boyce (2011) indicaron que al comparar los porcentajes de tiempo que niños y niñas gastan en actividad física, es evidente que los niños son más activos, independientemente de entorno. Contrario a las niñas de este estudio, quienes expresaron que el recreo es una gran oportunidad para socializar con las amigas, en el caso de los niños, ellos perciben el recreo como una gran oportunidad para jugar. Estos datos aportados por los escolares participantes en este estudio son congruentes con datos de Álvarez (2016) y Haug et al. (2010), los cuales agregan que lo anterior es independiente del nivel o grado de los observados.

Aunque las niñas de este estudio reportaron que las actividades preferidas son las relaciones sociales que involucran el hablar y caminar en grupos pequeños, ellas expresaron el deseo de involucrarse en actividades físicas, si existieran mejores condiciones. En relación con esta percepción que limita el ser más activas a las niñas, este es un elemento básico que debe ser atendido para garantizar el incremento de la actividad física en el entorno de los recreos escolares.

Evidentemente, se ha sugerido que los recreos podrían aportar hasta un $40 \%$ de la actividad física diaria recomendada sin embargo, en este estudio dicha situación no se cumple. Especialmente en el caso de las niñas los patrones de actividad física muestran o están ligados a patrones de movimiento dados por roles diferenciados entre género (Blaes et al., 2013).

La diferencia en relación con el género en cuanto a la actividad física moderada-vigorosa significativa encontrada en este estudio es consistente con estudios previos que utilizaron SOPLAY. Específicamente, los niños participaron en actividades vigorosas y las niñas participaron en actividades de naturaleza sedentarias (Willenberg et al., 2010). Aunque los niños fueron algo más activos que las niñas, las diferencias no son muy grandes. Tratando de entender la conducta más sedentaria o menos activa de las niñas se debe poner atención a lo expresado por ellas en este estudio. Las niñas de niveles superiores manifestaron que a ellas les gustan las actividades menos vigorosas o sedentarias, que involucran el grupo social de amigas, como hablar y caminar por las instalaciones de la escuela; sin embargo, las niñas de primer grado se mostraron atraídas por juegos de perseguir, como contagio y manifestaron que, cuando se presenta la oportunidad y los de niveles superiores les permiten, ellas participan.

Estudios en Inglaterra y Australia (Parrish, Yeatman, Iveson y Russell, 2012; Ridgers y Ridgers, Fairclough y Stratton, 2010) han mostrado que la percepción de seguridad que ofrece el ámbito escolar en el recreo para los niños y las niñas no presenta diferencias; sin embargo, los hallazgos de este estudio sí muestran que las niñas se sienten intimidadas en ambientes donde no se presenta la supervisión o presencia de las maestras. Este hecho se podría explicar tomando en cuenta que las niñas expresaron que ellas preferían el uso de espacios reducidos y conocidos que les permitiera socializar.

Como un punto importante dentro de los hallazgos de esta investigación está el hecho de que los estudiantes en escuelas con horario ampliado mostraron mayor cantidad de conductas sedentarias que los de las escuelas con horario alterno, lo cual ocasiona que se vuelva a considerar la infraestructura escolar como una barrera para que se involucren en actividad física. En las escuelas de horario ampliado todos los estudiantes tienen que compartir el mismo espacio de juego, contrario a las escuelas que alternan, en las que solo un ciclo está en un momento dado compartiendo el mismo espacio del recreo. Al respecto, se ha comentado que cuando existe mayor densidad de estudiantes en un entorno escolar, menores serán las posibilidades para estos de participar en actividades físicas, sobre todo para los más pequeños. Los estudiantes de este estudio manifestaron que definitivamente el compartir los espacios con niños de mayor edad los ponía en una desventaja a la hora de encontrar un espacio para jugar, lo cual los coloca también en desventaja para lograr los beneficios cognitivos, sociales, emocionales y físicos que ofrece la participación en actividad física en el entorno de recreo (American Academy of Pediatrics, 2013). Shen (2014) establece que el cambio de conducta está asociado con el ambiente físico y social apropiado, por lo que las limitaciones de espacio en las escuelas de horario ampliado podría ser un elemento a tomar en cuenta para entender los resultados de este estudio.

La percepción de la población infantil en este estudio ha revelado que las influencias del entorno social y organizacional han sido percibidas como las de mayor peso a la hora de involucrarse en actividad física 
durante los recreos escolares, lo cual hace indispensable que los programas o intervenciones escolares en la promoción de la actividad física contemplen, con mayor énfasis, estos factores.

\section{Conclusión}

A través de la utilización del Sistema de Observación (SOPLAY) se ha podido determinar que los niveles de actividad física realizada por los estudiantes en los recreos no contribuyen a lograr la hora diaria de actividad moderada-vigorosa recomendada por los organismos internacionales para los estudiantes de primaria. Las niñas son las que más lejos se encuentran de las recomendaciones. En relación con el tipo de escuela, con horario ampliado o alterno, se presenta mayor incidencia de conductas activas en las escuelas de horario ampliado.

En aras de comprender los hallazgos de este estudio, se debe prestar atención a la percepción de los niños en cuanto a las barreras para realizar actividad física durante el recreo, desde una perspectiva cualitativa. Se identificaron como barreras importantes para la realización de actividad física durante el recreo: la falta de instalaciones y equipos, la falta de políticas a nivel escolar, la ropa, la falta de apoyo de la maestra y la falta de espacio. Lo anterior indica que los ambientes físicos escolares tienen un impacto en el comportamiento, incluyendo la inclinación a participar en actividad física.

Este estudio enfatiza la necesidad de replantearse el papel de las escuelas para que lideren la promoción de la salud física y la prevención de la obesidad. El entender, desde la perspectiva de la población infantil, que los niveles percibidos con mayor influencia para realizar actividad física son el entorno social y físico indica que las estrategias en la promoción de la salud física que contribuyan a la salud integral de los escolares deben contemplar el trabajar para eliminar esas barreras, así como contribuir a incrementar la percepción de ellos hacia entornos que propician movimiento.

Con base en los resultados, se hace evidente que existe una gran necesidad de que los recreos sean conceptualizados como parte esencial en la formación integral de los niños, no solo por el estímulo esencial que reciben en su desarrollo físico y social, sino también por el efecto positivo en el rendimiento académico que reciben al jugar, por lo que se hace necesario el desarrollo de una política que declare al recreo como un momento para el juego de los escolares, donde los estudiantes encuentren las condiciones mínimas para disfrutar esos momentos lúdicos, que podría eventualmente aportar a en la formación de escolares más saludables, con maestras que comprenden el valor único de esos momentos para sus alumnos.

\section{Referencias}

Álvarez, C. (2016). Entendiendo los factores que determinan la actividad física en el entorno escolar desde la perspectiva de los niños y niñas. MHSALUD: Revista en Ciencias del Movimiento Humano y Salud, 13(1), 1-17. Recuperado de https://doi.org/10.15359/mhs.13-1.2

American Academy of Pediatrics. (2013). The crucial role of recess in school. Pediatrics, 131(1), 183-187. Recuperado de https://doi.org/10.1542/peds.2012-2993

Anthamatten, P., Brink, L., Lampe, S., Greenwood, E., Kingston, B. y Nigg, C. (2011). An assessment of schoolyard renovation strategies to encourage children's physical activity. International Journal of Behavioral Nutrition and Physical Activity, 8(27), 2-9. Recuperado de https:// doi.org/10.1186/1479-5868-8-27

Arias, E. (2014). Niveles de actividad física de niños y adolescentes durante el descanso en la escuela, un estudio observacional con el uso de SOPLAY. Revista Educación Física y Deportes, 33(1), 175-191. Recuperado de http://aprendeenlinea.udea.edu.co/revistas/index.php/ educacionfisicaydeporte/article/viewFile/20415/17246 
Biddle, S.J.H. y Asare, M. (2011). Physical activity and mental health in children and adolescents: a review of reviews. British Journal of Sports Medicine, 45(11), 886-895. Doi: https://doi.org/10.1136/ bjsports-2011-090185

Blaes, A., Ridgers, N.D., Aucouturier, J., Van Praagh, E, Berthoin, S. y Baquet, G. (2013). Effect of a playground makings intervention on school recess physical activity in French children. Preventive Medicine, 57(5), 580-584. Doi: https://doi.org/10.1016/j.ypmed.2013.07.019

Davison, K. y Lawson, C. (2006). Do attributes in the physical environment influence children's physical activity? A review of the literature. International Journal of Behavioral Nutrition and Physical Activity, 3(19), 1-17. Doi https://doi.org/10.1186/1479-5868-3-19

Elliot, S. Combs S. y Boyce, R. (2011). Recess physical packs in elementary schools: a qualitative investigation. The Physical Educator, 68(3), 150-162. Recuperado de http://js.sagamorepub.com/pe/ article/view/2264

Frago, J., Murillo, B., García, L, Aibar, A. y Zaragoza, J. (2017). Physical Activity Levels during unstructured recess in spanish primary and secondary schools. European Journal of Human Movement, 38, 40-52. Recuperado de https://dialnet.unirioja.es/servlet/articulo?codigo=6066036

Fuster, V. (2014). Top 10 cardiovascular therapies and interventions for the next decade. Nature reviews. Cardiology, 11(11), 671-683. Doi: https://doi.org/10.1038/nrcardio.2014.137

Janssen, I., y Leblanc, A. G. (2010). Systematic review of the health benefits of physical activity and fitness in school-aged children and youth. International Journal of Behavioral Nutrition and Physical Activity, 7(40), 1-16. Doi: https://doi.org/10.1186/1479-5868-7-40

Haug, E., Torsheim, T., Sallis, J.F. y Samdal, O. (2010). The characteristics of the outdoor school environment associated with physical activity. Health Education Research, 25(2), 248-256. Doi: https://doi.org/10.1093/her/cyn050

Hayman, L. L., Williams, C. L., Daniels, S. R., Steinberger, J., Paridon, S., Dennison, B., McCrindle, B.W., \& Committee on Atherosclerosis, Hypertension, and Obesity in Youth (AHOY) of the Council on Cardiovascular Disease in the Young, American Heart Association. (2004). Cardiovascular health promotion in the schools: A statement for health and education professionals and child health advocates from the Committee on Atherosclerosis, Hypertension and Obesity in Youth (AHOY) of the Council on Cardiovascular Disease in the Young, American Heart Association. Circulation, 110, 2266-2275. Doi: https://doi.org/10.1161/01.CIR.0000141117.85384.64

Ickes, M., Erwin, H. y Beighle, A. (2013). Systematic Review of Recess Interventions to increase Physical Activity. Journal of Physical Activity and Health, 10(6), 910-926. PMID: 23074100. Doi: https:// doi.org/10.1123/jpah.10.6.910

Instituto de Nutrición de Centroamérica y Panamá [INCAP]. (2016). Situación de la obesidad en Centroamérica y República Dominicana. Revista A.C. Nota Técnica, 1-7. Recuperado de http://www.incap.int/sisvan/index.php/es/cooperacion-tecnica-en-la-region/ documentos-especializados/doc_view/279-nota-tecnica-situacion-de-la-obesidad-en-centroamerica-y-republica-dominicana-2016

Ishii, K., Shibata, A., Sato, M. y Koichiro O. (2014). Recess Physical Activity and Perceived School Environment among Elementary School Children. Journal of Environmental Research and Public Health, 11(7), 7195-7206. Doi: https://doi.org/10.3390/ijerph110707195

Mays, A., Graber, K. y Daum, D. (2012). Children's recess physical activity: Movement Patterns and Preferences. Journal of Teaching in Physical Education, 31(2), 146-162. Doi. https://doi.org/10.1123/ jtpe.31.2.146

McKenzie, T.L., Marshall, S.J., Sallis, J.F. y Conway, T.L. (2000). Leisure-time physical activity in school environments: an observational study using SOPLAY. Prevention Medicine, 30(1), 70-77. Recuperado de https://doi.org/10.1006/pmed.1999.0591 
Mckenzie, T.L., Sallis, J.F., \& Nader, P.R.(1991). System for observing fitness instruction time. Journal of Teaching in Physical Education, 11, 195-205.

Ministerio de Salud y Ministerio de Educación Pública. (2016). Censo Escolar Peso/Talla 2016. Resultados. Recuperado de http://www.mep.go.cr/sites/default/files/page/adjuntos/resultados-censo-escolarpeso-talla-2016.pdf

Organización Mundial de la Salud [OMS]. (2010). Recomendaciones Mundiales sobre Actividad Física para la Salud. Recuperado de http:// http://apps.who.int/iris/ bitstream/10665/44441/1/9789243599977_spa.pdf

Organización Panamericana de la Salud [OPS]. (2010). Encuesta Mundial de Salud Estudiantil. [Global School-based Student Health Survey (GSHS)]. Recuperado de http://www.paho.org/hq/index.php? option=com_c\%20ontent\&view=article\&id=11655\%3Agshs\&catid=8472\%20\%3Agshs-data\&lang=en $\% 20$

Ozdemir, A. y Yilmaz, O. (2008). Assessment of outdoor school environments and physical activity in Ankara's primary schools. Journal of Environmental Psychology, 28 (3), 287-300. Doi: https:// doi.org/10.1016/j.jenvp.2008.02.004

Parrish, A., Yeatman, H., Iveson, D. y Russell, K. (2012). Using Interviews and Peer Pairs to Better Understand how School environments Affect Yong Children Playground Physical Activity Levels: a Qualitative Study. Health Education Research, 27(2), 269-280. Doi: https://doi.org/10.1093/her/ cyr049

Patton, M.Q. (2002). Qualitative Research and Evaluation Method. Integrating Theory and Practice. (4th ed.). Thousand Oaks, CA: Sage.

Pawlowski, C., Tjørnhøj-Thomsen T., Shipperijn, J. y Troelsen, J. (2014). Barriers for Recess Physical Activity: A Gender Specific Focus group Exploration. BMC Public Health, 14, 3-10. Doi: https:// doi.org/10.1186/1471-2458-14-639

Pereira, Z. (2011). Los diseños de método mixto en la investigación en educación: Una experiencia concreta. Revista Electrónica Educare, 15(1), 15-29. Recuperado de http://revistas.una.ac.cr/ index.php/EDUCARE/article/viewFile/867/793

Pitney, W. y Parker, J. (2009). Qualitative Research in Physical Activity and the Health Professions. Champaign, IL: Human Kinetics.

Ridgers, N.D., Fairclough, S.J. y Stratton, G. (2010). Variables associated with children's physical activity levels during recess: The A-CLASS project. International Journal of Behavioral Nutrition and Physical Activity, 7(74), 1-8. Doi: https://doi.org/10.1186/1479-5868-7-74

Ridgers, N. y Stratton, G. (2005). Physical Activity during school recess: The Liverpool Sporting Playground Project. Pediatric Exercise Science, 17, 281-290. Recuperado de https://doi.org/10.1123/pes.17.3.281

Sallis, J.F., Conway, T.L., Prochaska, J.J., McKenzie, T.L., Marshall, S.J. y Brown, M. (2001). The association of school environments with youth physical activity. American Journal of Public Health, 91(4), 618-620.

Springer, A., Tanguturi, Y., Ranjit, N., Skala, K. y Kelder, S. (2013). Physical Activity During Recess in Low-Income Third-Grade Texas Students. American Journal of Health Behavior, 37(3), 318-324. doi: 10.5993/AJHB.37.3.4 Doi: https://doi.org/10.2105/AJPH.91.4.618

Shen, B. (2014) Outside-school physical activity participation and motivation in physical education. British Journal of Educational Psychology, 84(1), 40-57. Doi: https://doi.org/10.1111/bjep.12004

Verstraete, S. J., Cardon, G. M., De Clercq, D. L. y De Bourdeaudhuij, I. M. (2006). Increasing children's physical activity levels during recess periods in elementary schools: the effects of providing game equipment. European Journal of Public Health, 16(4), 415-419. Recuperado de https:// doi.org/10.1093/eurpub/ckl008 
Ward, D., Saunders, R. y Pate, R. (2007). Physical Activity Interventions in Children and Adolescents. USA: Human Kinetics Publishers. Recuperado de https://doi.org/10.1016/j.jsams.2009.02.011

Willenberg, L.J., Ashbolt, R., Holland, D., Gibbs, L., MacDougall, C., Garrard, J., Green, J. y Waters, E. (2010). Increasing school playground physical activity: a mixed methods study combining environmental measures and children's perspectives. Journal of Science and Medicine in Sport, 13(2), 210-216. Recuperado de https://doi.org/10.1016/j.jsams.2009.02.011

CC BY-NC-ND 